\title{
Interdisciplinary COPD intervention in primary care: a cluster randomised controlled trial
}

\author{
Jenifer Liang (10), Michael J. Abramson (10) ${ }^{2}$, Grant Russell ${ }^{3}$, Anne E. Holland (10 ${ }^{4}$, \\ Nicholas A. Zwar ${ }^{5,6}$, Billie Bonevski ${ }^{7}$, Ajay Mahal ${ }^{8}$, Paula Eustace $\mathbb{1}^{9}$, \\ Eldho Paul ${ }^{2}$, Kirsten Phillips ${ }^{10}$, Narelle S. Cox ${ }^{11}$, Sally Wilson ${ }^{1,12}$ and \\ Johnson George (1) ${ }^{1,2}$
}

@ERSpublications

An interdisciplinary model of care for COPD in primary care involving smoking cessation support, home medicines review, and home-based pulmonary rehabilitation did not demonstrate superiority over usual care; low uptake by GPs and patients was a challenge http://ow.ly/DmkC30nxL9D

Cite this article as: Liang J, Abramson MJ, Russell G, et al. Interdisciplinary COPD intervention in primary care: a cluster randomised controlled trial. Eur Respir J 2019; 53: 1801530 [https://doi.org/10.1183/ 13993003.01530-2018].

ABSTRACT We evaluated the effectiveness of an interdisciplinary, primary care-based model of care for chronic obstructive pulmonary disease (COPD).

A cluster randomised controlled trial was conducted in 43 general practices in Australia. Adults with a history of smoking and/or COPD, aged $\geqslant 40$ years with two or more clinic visits in the previous year were enrolled following spirometric confirmation of COPD. The model of care comprised smoking cessation support, home medicines review (HMR) and home-based pulmonary rehabilitation (HomeBase). Main outcomes included changes in St George's Respiratory Questionnaire (SGRQ) score, COPD Assessment Test (CAT), dyspnoea, smoking abstinence and lung function at 6 and 12 months.

We identified 272 participants with COPD (157 intervention, 115 usual care); 49 (31\%) out of 157 completed both HMR and HomeBase. Intention-to-treat analysis showed no statistically significant difference in change in SGRQ at 6 months (adjusted between-group difference 2.45 favouring intervention, 95\% CI -0.89-5.79). Per protocol analyses showed clinically and statistically significant improvements in SGRQ in those receiving the full intervention compared to usual care (difference 5.22, 95\% CI 0.19-10.25). No statistically significant differences were observed in change in CAT, dyspnoea, smoking abstinence or lung function.

No significant evidence was found for the effectiveness of this interdisciplinary model of care for COPD in primary care over usual care. Low uptake was a limitation.

This article has supplementary material available from erj.ersjournals.com

Received: Aug 132018 | Accepted after revision: Jan 252019

This study was registered at www.anzctr.org.au/ with identifier number ACTRN12614001155684. Anonymous patient level data, technical appendix and statistical code are available on reasonable request from the corresponding author, but may require ethics approval.

Copyright OERS 2019 


\section{Introduction}

Chronic obstructive pulmonary disease (COPD) is a major public health problem. Symptoms and complications can greatly impair patients' ability to perform activities of daily living and compromise quality of life.

Management of COPD involves multiple health professionals for delivery of both pharmacological and nonpharmacological interventions for optimal outcomes. The roles of the general practitioner (GP) and other healthcare professionals in the management of COPD are important [1]. There is a trend towards an interdisciplinary approach to COPD management whereby team members from different disciplines work collaboratively, with a common aim, to set goals, make decisions and share resources and responsibilities [2]. The team of health professionals from different disciplines work together with the patient and undertake assessment, diagnosis, intervention, goal-setting and the development of a care plan [3]. Integrated disease management requires multidisciplinary input and may be further enhanced with an interdisciplinary approach. Integrated disease management is "a group of coherent interventions designed to prevent or manage one or more chronic conditions using a systematic, multidisciplinary approach and potentially employing multiple treatment modalities" [4]. A recent Cochrane review of multi-component, multiprofessional, integrated disease management programmes for COPD showed positive effects on disease-specific quality of life, exercise capacity, hospital admissions and length of hospital stay, but not on dyspnoea or lung function [5]. Additionally, integrated disease management programmes have demonstrated positive effects on level of follow-up, pulmonary rehabilitation attendance, self-reported daily activity and disease knowledge $[6,7]$. However, recent multi-professional interventions for COPD management in primary care have shown no additional benefit beyond usual care on health-related quality of life (HRQoL) $[6,8,9]$.

Underutilisation of spirometry for diagnosis of COPD, suboptimal pharmacological treatment and low referral rates for pulmonary rehabilitation are common challenges in COPD management [10-13]. Smoking cessation remains crucial in COPD prevention and management, with up to $50 \%$ of smokers developing clinically significant COPD [14]. Medication adherence and incorrect inhaler technique are known issues in the COPD population $[15,16]$. Pharmacist-led interventions in the community setting, involving medication review and patient education have shown positive impact on inhaler technique and medication adherence [17, 18]. The home medicines review (HMR) service is currently a governmentfunded service in Australia for patients with an identifiable clinical need, at risk of medication misadventure and/or on multiple medications, including inhaler devices. Although benefits of pulmonary rehabilitation in COPD management are well established, access and completion of these programmes remain low internationally [19-21]. Home-based pulmonary rehabilitation programmes have been evaluated and shown to provide similar outcomes to conventional centre-based rehabilitation [22].

Novel models of care aimed at improving smoking cessation, COPD diagnosis, symptom control and exacerbation prevention through interdisciplinary interventions may enhance quality of life. To date, multi-professional programmes for COPD in primary care have not had explicit regard to the interaction among health professionals delivering smoking cessation support, HMRs and home-based pulmonary rehabilitation. We designed an interdisciplinary consumer-centred intervention where GPs, clinic staff, pharmacists and physiotherapists worked collaboratively, with a common purpose, to set goals, make decisions and share resources and responsibilities in the delivery of care.

\section{Aim}

The primary aim was to implement an interdisciplinary model of care (Review of Airway Dysfunction and Interdisciplinary Community-Based Care of Adult Long-Term Smokers; RADICALS) in Australian primary care and evaluate its effectiveness on HRQoL at 6 months.

Affiliations: ${ }^{1}$ Centre for Medicine Use and Safety, Faculty of Pharmacy and Pharmaceutical Sciences, Monash University, Melbourne, Australia. ${ }^{2}$ Dept of Epidemiology and Preventive Medicine, School of Public Health and Preventive Medicine, Monash University, Melbourne, Australia. ${ }^{3}$ Southern Academic Primary Care Research Unit, Dept of General Practice, Monash University, Melbourne, Australia. ${ }^{4}$ Discipline of Physiotherapy, La Trobe University, Alfred Health, and Institute for Breathing and Sleep, Melbourne, Australia. ${ }^{5}$ School of Public Health and Community Medicine, University of New South Wales, Sydney, Australia. ${ }^{6}$ Faculty of Health Sciences and Medicine, Bond University, Gold Coast, Australia. ${ }^{7}$ School of Medicine and Public Health, University of Newcastle, Newcastle, Australia. ${ }^{8}$ The Nossal Institute for Global Health, University of Melbourne, Melbourne, Australia. ${ }^{9}$ Eastern Melbourne PHN (EMPHN), Melbourne, Australia. ${ }^{10}$ Lung Foundation Australia (LFA), Milton, Australia. ${ }^{11}$ Discipline of Physiotherapy, La Trobe University and Institute for Breathing and Sleep, Melbourne, Australia. ${ }^{12}$ Dept of Infrastructure Engineering. The University of Melbourne, Melbourne, Australia.

Correspondence: Johnson George, Centre for Medicine Use and Safety, Faculty of Pharmacy and Pharmaceutical Sciences, Monash University, 381 Royal Parade, Parkville, VIC 3052, Australia. E-mail: johnson.georgelamonash.edu 
We hypothesised that a primary care-based interdisciplinary team, comprising the patient's GP, clinic staff, pharmacist and physiotherapist, delivering a model of care involving smoking cessation support, HMR and home-based pulmonary rehabilitation would improve COPD-related HRQoL compared to usual care at 6 months.

Secondary objectives were to determine the uptake of this novel model of care, improve the diagnosis of COPD in those at risk in primary care, assist smokers to quit and improve symptoms, psychosocial outcomes (anxiety and depression) and lung function of those with COPD at 6 and 12 months.

\section{Methods}

We performed a two-arm, cluster randomised controlled trial. Cluster randomisation minimises the risk of contamination across intervention and usual care groups. The detailed protocol of the trial and baseline cohort characteristics are available elsewhere [13, 23]. This article focuses on the effectiveness of RADICALS on primary and secondary outcomes at 6 and 12 months. The trial was registered with the Australia and New Zealand Clinical Trials Registry (identifier number ACTRN12614001155684).

\section{Clinic recruitment and randomisation}

In brief, group or solo GP clinics with $\geqslant 1000$ patients on their databases were approached directly or with assistance from primary care organisations. After obtaining signed agreement, clinics were block randomised (block sizes of four and six) to usual care or intervention arms using an externally managed web-based randomisation programme. Clinics were notified of their allocation. A baseline survey was completed by clinics to provide basic data, including details related to practice staff, availability of spirometers and staff training undertaken previously.

\section{Patient selection and data collection}

Trained research assistants at each clinic identified potential participants by searching patient databases and contacting them via mail or telephone. Patients were eligible if they were aged $\geqslant 40$ years, had at least two clinic visits during the previous year and self-reported being a current/ex-smoker $(\geqslant 10$ pack-year smoking history) or those who had a documented diagnosis of COPD on clinic records or were being treated with COPD-specific medications.

Participants attended a baseline interview at their clinic after providing written consent. Data collection was undertaken, and case-finding used the hand-held COPD- $6^{\circ}$ device (Vitalograph, Ennis, Ireland) [24]. Those with forced expiratory volume in $1 \mathrm{~s}(\mathrm{FEV} 1)$ /forced expiratory volume in 6s (FEV6) $<0.75$ [25] were referred for spirometry (Easy on-PC spirometers; ndd Medizintechnik AG, Zürich, Switzerland). Spirometry was performed according to American Thoracic Society/European Respiratory Society guidelines [26]. A post-bronchodilator FEV1/forced vital capacity $<0.7$ suggested COPD [27]. Recent spirometry undertaken outside the trial was assessed, if accessible. An algorithm guided research assistants in establishing a diagnosis of COPD (available from authors). Uncertain cases were interpreted by a respiratory scientist and/or respiratory physician. Spirometry results, modified Medical Research Council (mMRC) and COPD Assessment Test (CAT) scores were communicated to each participant's GP/clinic staff for review and confirmation of diagnosis with the patient. Only those participants diagnosed as having COPD were included in the primary effectiveness analysis.

\section{Usual care}

GPs in usual care practices continued to provide routine care to their patients. Copies of the COPD-X Plan [28] and the Smoking Cessation guide [29] were provided to clinic staff. Spirometry results and interpretation were made available for GPs to review. Participants were given the Lung Foundation Australia booklet "Better Living with Chronic Obstructive Pulmonary Disease - A Patient Guide" [30]. Quitline referral was provided to those who were smokers.

\section{Intervention}

The RADICALS model of care was underpinned by Australian COPD-X guidelines [27].

In addition to usual care, GPs and other staff from intervention group practices were offered training on spirometry and the COPD-X guidelines [28], and a smoking cessation guide [29].

The RADICALS model of care comprised the following interventions:

Individualised smoking cessation support was provided to smokers using QUIT resources and guided by a treatment algorithm [31]. This consisted of brief counselling and Quitline referral provided by research assistants during baseline interview to all smokers in the intervention arm, regardless of COPD diagnosis. Over-the-counter and/or prescription medications for smoking cessation were also recommended, if appropriate. 
The HMR [32] was performed by an accredited consultant pharmacist and consisted of an interview with participants in their homes (duration $\sim 1.5 \mathrm{~h}$ ). The interview allowed the pharmacist to assess medication use and participants' knowledge about their medications (including inhaler use and technique), provide education focusing on medication use and further individualised smoking cessation support, if relevant. A report was subsequently generated by the pharmacist and forwarded to the participant's GP outlining recommendations for optimising medication use, especially for COPD.

The 8-week home-based pulmonary rehabilitation (HomeBase) programme [22], delivered by a specifically trained physiotherapist, consisted of one home visit and weekly follow-up telephone calls. Home-based aerobic and resistance exercise training was individually prescribed. Telephone calls employed motivational interviewing principles to achieve disease-specific self-management training and exercise progression.

The model of care was coordinated by the research assistant at each site under the supervision of each participant's GP and clinic staff. Consenting patients were referred by the GP, at their discretion, to HomeBase and HMR. Following real-world practice, normal processes of spirometry results review and patient consent for service referrals occurred.

\section{Follow-up}

Participants were followed at 6 and 12 months after baseline by research assistants blinded to clinic and group allocation. Follow-ups (telephone and/or face-to-face) involved completion of a structured questionnaire with outcomes of interest, post-bronchodilator spirometry testing and exhaled carbon monoxide (CO) testing in smokers (if self-reported not smoking in the previous 7 days).

\section{Outcomes and measurements}

The primary outcome was change in the overall HRQoL, measured using St George's Respiratory Questionnaire (SGRQ) [33] score, at 6 months from baseline.

Secondary outcomes measured in participants at 6 and 12 months from baseline included changes in 1) SGRQ score [33] (at 12 months); 2) CAT score [34]; 3) mMRC grade [35, 36]; 4) lung function (FEV1 \% predicted) [37]; 5) Hospital Anxiety and Depression Scale (HADS) score [38, 39]; 6) Heaviness of Smoking Index (HSI) score [40]; and 7) proportions of COPD participants with biochemically verified 7-day point prevalence smoking abstinence: exhaled CO levels were measured using a handheld piCO Smokerlyzer (Bedfont Scientific, Maidstone, UK) to confirm self-report of 7-day point prevalence abstinence (a participant with $\mathrm{CO}$ level $\leqslant 6 \mathrm{ppm}$ was considered abstinent; missing data for smoking-related outcomes were treated in accordance with the Russell standard [41], whereby a smoker lost to follow-up was considered to have continued to smoke).

Process outcomes including the uptake of the HMR and HomeBase by GPs and participants and inhaler-related issues identified during HMR were measured by reviewing participant logs and notes recorded by the HMR pharmacist and HomeBase physiotherapists delivering these services.

Description of each outcome measure is included in the published study protocol [23].

\section{Sample size}

Change in SGRQ score at 6 months from baseline was the primary effectiveness end-point. A difference of at least four points in SGRQ between treatment arms was considered clinically significant [33]. Assuming a standard deviation of 10 points in SGRQ, 99 participants per group ( $80 \%$ power, $\alpha=0.05$ ) were needed to detect this. The required sample was 108 per arm (intraclass correlation=0.01 [42], cluster size $=10$ ). At least 28 primary care practices needed to be recruited and 14 each randomised to intervention and usual care arms. Further details have been published previously [23].

\section{Statistical analysis}

Analyses were conducted in accordance with a predefined analysis plan [23]. Baseline characteristics of the intervention and control groups were summarised according to data type and distribution.

Outcomes were assessed at the participant level. All regression analyses were adjusted for clustering, age, education, income, current smoking status and prior COPD diagnosis. The effectiveness analysis was according to an intention-to-treat principle. Mean change in SGRQ score at 6 months in each treatment group was estimated. Differences between groups and the corresponding $95 \%$ confidence intervals were determined. Multivariable analysis was performed using multiple linear regression, adjusting for baseline imbalances and confounders. Multiple imputation was generated for missing data based on the assumption that data were from a multivariate normal distribution and were missing at random. The regression method was used for imputation with 10 imputed datasets used for each variable. 
Per-protocol analyses (predefined) were conducted to determine effectiveness within participants completing the intervention as intended. Completion of HomeBase was defined as completion of a minimum of $70 \%$ of total sessions (i.e. at least six out of eight sessions in the programme) [22]. Completion of HMR was defined as having been present at a pre-booked appointment and participating in an interview with the consultant pharmacist. Therefore, intervention group participants were categorised according to their degree of completeness: "full intervention" (completion of both HMR and HomeBase), "partial intervention" (HMR only, HomeBase only, partial HomeBase only or HMR and partial HomeBase) or "no intervention". Baseline participant characteristics were compared between 1) those who completed the full intervention and those who did not; and 2) those who completed the trial and those who were lost to follow-up. Differences in baseline clinic characteristics were determined for those clinics with at least one participant completing the full intervention and those clinics with no full intervention completers.

All analyses were performed using Statistical Package for Social Sciences (SPSS) (version 24.0; IBM, Armonk, NY, USA) or Stata version 14.0 (StataCorp, College Station, TX, USA).

\section{Results}

43 clinics were randomised to intervention $(n=21)$ or usual care $(n=22)$; two clinics randomised to the usual care arm withdrew before recruiting any participants (figure 1). Characteristics of clinics have been described previously [13]. Between February 2016 and April 2017, 1050 participants were recruited (618 from intervention clinics and 432 from usual care clinics), of whom 272 were confirmed to have COPD (157 from intervention clinics and 115 from usual care clinics). At baseline, the groups appeared similar, although intervention group participants were older and more likely to have a trade rather than university education, but less likely to be current smokers than the usual care group (table 1).

The drop-out rates at 12-month follow-up were similar (intervention group 44 (28\%) out of 157 compared to $38(33 \%)$ out of 115 in usual care, $\mathrm{p}=0.45)$. Participants who completed the 12 -month follow-up were older, more likely to be in a relationship, not living independently and had better lung function (i.e. higher FEV1 \% pred) compared to those who dropped out (supplementary file).

\section{Primary outcome}

SGRQ scores improved in both groups between baseline and 6 months; however only the change in SGRQ score within the intervention group was statistically significant (3.07, 95\% CI 0.73-5.42) (table 2). Difference in SGRQ score changes between groups was not statistically significant (adjusted mean difference 2.45 favouring intervention, 95\% CI -0.89-5.79) at 6 months (table 2). Multiple imputation analyses performed for missing SGRQ data showed similar results (supplementary table S1). No statistically significant differences in proportions of participants achieving the minimum clinically important difference (MCID) in SGRQ of four points or more were observed between groups (intervention 47 (44.8\%) out of 105; usual care 38 (42.2\%) out of 90; data not shown).

\section{Secondary outcomes \\ 6-month outcomes}

Changes in CAT and lung function (FEV1 \% pred) from baseline at 6 months between groups were not statistically significant (table 3). There were no differences in HSI scores (median 3, interquartile range 2-4 in both groups; $\mathrm{p}=0.62$ ) and proportion of smokers with biochemically verified 7-day point prevalence smoking abstinence (intervention six (7.1\%) out of 84 , usual care three $(3.7 \%)$ out of $82 ; \mathrm{p}=0.50$ ) between groups at 6 months. Multiple imputation analyses performed for missing CAT and FEV1 \% pred data showed similar results (supplementary table S1). Proportions of participants who showed improvements (from baseline) in HADS scores and mMRC grades were not significantly different between groups (table 3 ).

\section{2-month outcomes}

No statistically significant differences were observed between groups in change in SGRQ, CAT, proportions of participants achieving SGRQ MCID (data not shown) and those showing improvements (from baseline) in HADS anxiety/depression scores and mMRC grades (tables 2 and 3). Multiple imputation analyses performed for missing SGRQ and CAT outcome data showed similar results (supplementary table S1). Spirometry was repeated only in those attending a face-to-face follow-up interview (6\% of participants); smoking abstinence outcomes were restricted to those reporting abstinence at 6 months.

Process outcomes

Uptake of the intervention was poor. Only 31\% (49 out of 157) completed the full intervention, a quarter (26\%, 41 out of 157) partially completed the intervention (HMR only: 19 out of 40; HomeBase programme only: six out of 40; HMR and partially completed HomeBase programme: 13 out of 40; partially completed 


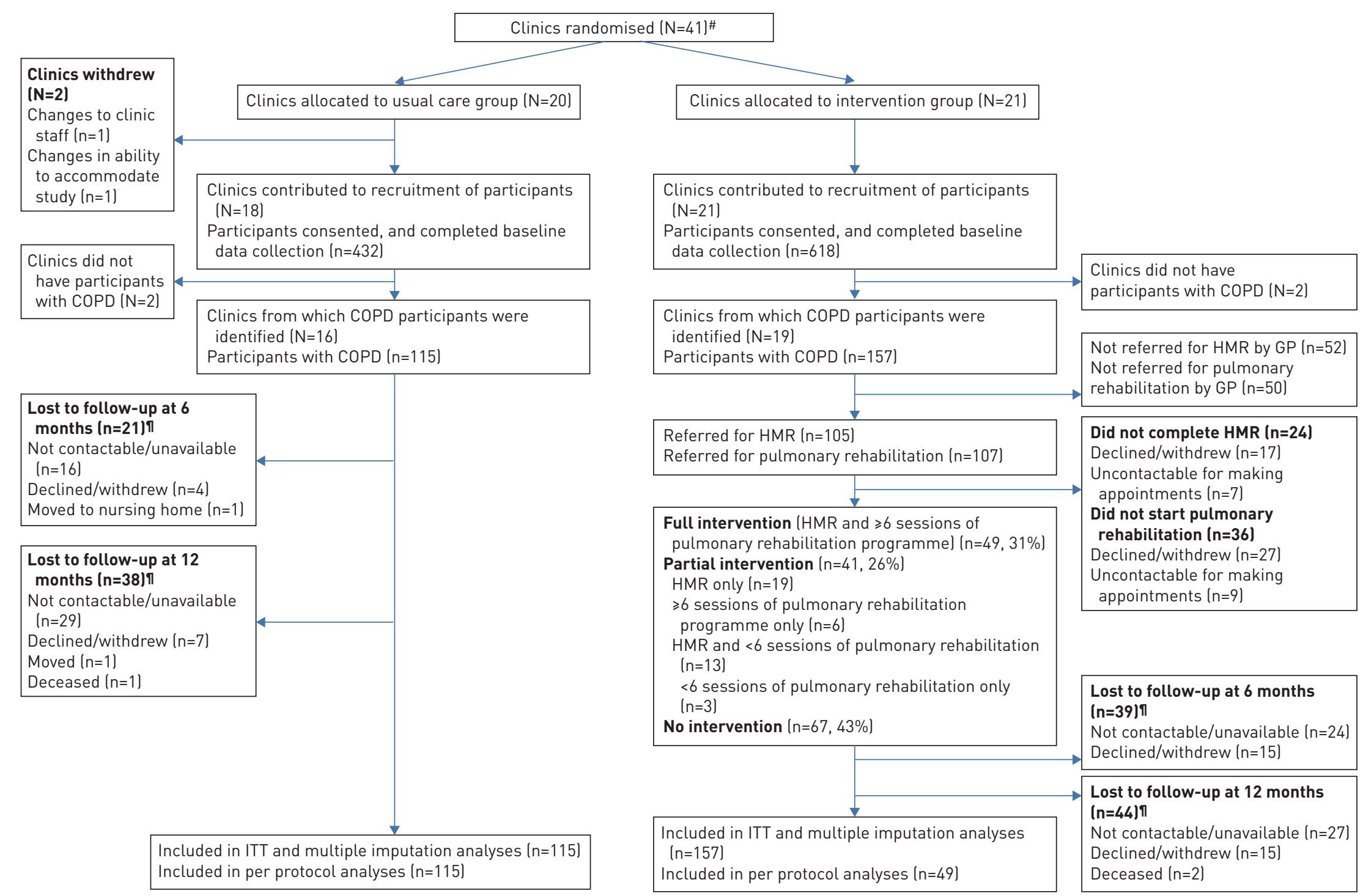

FIGURE 1 Review of Airway Dysfunction and Interdisciplinary Community-Based Care of Adult Long-Term Smokers (RADICALS) study flow diagram. N: numbers of clinics; n: numbers of participants; COPD: chronic obstructive pulmonary disease; GP: general practitioner; HMR: home medicines review; ITT: intention to treat. \#: Total of 43 clinics; two clinics belonged to the same consortium where GPs and clinic staff worked across clinics; these clinics were collectively treated as one entity and allocated to the same arm as the clinic recruited initially; ף: some participants were available at 6-month follow-up, but not available at 12-month follow-up, and vice versa. 
TABLE 1 Baseline demographic and clinical characteristics

\begin{tabular}{|c|c|c|}
\hline & Usual care & Intervention \\
\hline Subjects & 115 & 157 \\
\hline Clinics & 16 & 19 \\
\hline Age years & $61.7 \pm 10.1$ & $66.6 \pm 10.8$ \\
\hline Male & $72(62.6)$ & $95(60.5)$ \\
\hline Born in Australia" & $86(74.8)$ & $114(74.0)$ \\
\hline Mainly speak English at home & $113(98.3)$ & $154(98.1)$ \\
\hline \multicolumn{3}{|l|}{ Education" } \\
\hline No formal schooling/up to primary school/primary school & $4(3.5)$ & $14(9.1)$ \\
\hline High school & $63(54.8)$ & $68(44.2)$ \\
\hline Technical/further education & $22(19.1)$ & $51(33.1)$ \\
\hline University education/postgraduate & $26(22.6)$ & $21(13.6)$ \\
\hline \multicolumn{3}{|l|}{ Employment status" } \\
\hline Employed-full/part-time/casual & 25 (22.3) & $51(32.5)$ \\
\hline Retired/pensioner & $68(60.7)$ & 84 (53.5) \\
\hline Unemployed/home duties/student/unable to work/disabled & $19(17.0)$ & $22(14.0)$ \\
\hline \multicolumn{3}{|l|}{ Marital status" } \\
\hline Married/de-facto/engaged & $53(46.1)$ & $69(44.8)$ \\
\hline Separated/divorced/widowed/never married/single & $61(53.0)$ & $85(55.2)$ \\
\hline Undisclosed & $1(0.9)$ & $0(0.0)$ \\
\hline \multicolumn{3}{|l|}{ Current living arrangements ${ }^{\#}$} \\
\hline With family/friends/spouse/partner & $76(66.1)$ & $97(63.0)$ \\
\hline Alone at home & 36 (31.3) & $51(33.1)$ \\
\hline $\begin{array}{l}\text { Shared accommodation/community housing/refuge accommodation/ } \\
\text { residential facility/residential aged care facility }\end{array}$ & $3(2.6)$ & 6 (3.9) \\
\hline \multicolumn{3}{|l|}{ Average annual household income AUD ${ }^{1,+}$} \\
\hline$<30000$ & $45(40.2)$ & $74(49.7)$ \\
\hline $30000-59999$ & $25(22.3)$ & 32 (21.5) \\
\hline$\geqslant 60000$ & $18(16.1)$ & $31(20.8)$ \\
\hline Did not want to disclose & $24(21.4)$ & $12(8.1)$ \\
\hline Previously managed as having COPD & $56(48.7)$ & $74(47.1)$ \\
\hline Currently smoking & 82 (71.3) & $84(53.5)$ \\
\hline \multicolumn{3}{|l|}{ Heaviness of Smoking Index score (current smokers only) \} ^ { \S } $\\
{\hline \text { Low nicotine dependence (score } 0-2 \text { ) }} &{24(29.3)} &{21(26.3)} \\
{\hline \text { Moderate nicotine dependence (score 3-4) }} &{46(56.1)} &{44(55.0)} \\
{\hline \text { High nicotine dependence (score 5-6) }} &{12(14.6)} &{15(18.8)} \\
{\hline \text { Post-bronchodilator FEV1 \% predicted }{ }^{\#}} &{70.8 \pm 19.3} &{69.0 \pm 20.5} \\
{\hline \text { Post-bronchodilator FEV1/FVC" }} &{0.57 \pm 0.10} &{0.57 \pm 0.13} \\
{\hline \multicolumn{3}{|l|}{\text { Disease severity } \#, f}\text { Disease severity } \# , f} \\
{\hline \text { Mild }} &{82 \text { (71.9) }} &{108(69.7)} \\
{\hline \text { Moderate }} &{26(22.8)} &{30(19.4)} \\
{\hline \text { Severe }} &{6(5.3)} &{17(11.0)} \\
{\hline \multicolumn{3}{|l|}{\text { mMRC dyspnoea grade } \# \#}\text { mMRC dyspnoea grade } \# \#} \\
{\hline 0} &{39 \text { (34.2) }} &{37(23.6)} \\
{\hline 1} &{42(36.8)} &{70(44.6)} \\
{\hline 2} &{22 \text { (19.3) }} &{26(16.6)} \\
{\hline 3} &{10(8.8)} &{21(13.4)} \\
{\hline 4} &{1(0.9)} &{3(1.9)} \\
$\hline}
\end{tabular}

Data are presented as $\mathrm{n}$, mean \pm SD or $\mathrm{n}(\%)$. COPD: chronic obstructive pulmonary disease; FEV1: forced expiratory volume in $1 \mathrm{~s}$; FVC: forced vital capacity; mMRC: modified Medical Research Council. \#: missing data $n=3$; ๆ : missing data $n=11 ;{ }^{+}$: Australian annual pension rate for singles is $\sim A U D 24000 ;{ }^{\S}$ : missing data $\mathrm{n}=4 ;{ }^{f}$ : severity of COPD based on COPD-X plan: FEV $1 \%$ pred values $160-80 \%$ pred $=$ mild, $40-59 \%$ pred=moderate, $<40 \%$ pred=severe) [28]; 80 participants had $\mathrm{FEV} 1$ values $>80 \%$ pred, but had characteristic symptoms of mild COPD; ${ }^{\# \#}$ : missing data $n=1$.

HomeBase programme only: three out of 40) and 67 (43\%) participants did not receive any part of the intervention. Reasons for not receiving the intervention included GP non-referral, participant uncontactable for appointments, declining exercise, lack of perceived need for HMR, being too busy and declining due to personal/family circumstances. Of the COPD participants referred and eventually completing the HMR, 35 (43\%) out of 81 were assessed as having suboptimal inhaler technique and 70 (86\%) out of 81 were provided with a demonstration of their inhaler devices by the visiting HMR pharmacist. 
TABLE 2 St George's Respiratory Questionnaire (SGRQ) score differences from baseline to 6 and 12 months (intention-to-treat analysis)

\begin{tabular}{|c|c|c|c|c|c|c|c|c|}
\hline & \multicolumn{2}{|c|}{ Baseline } & \multicolumn{2}{|c|}{$\begin{array}{l}\text { Within-group change } \\
\text { at } 6 \text { months }\end{array}$} & \multirow{2}{*}{$\begin{array}{c}\text { Between-group } \\
\text { difference at } \\
6 \text { months }\end{array}$} & \multicolumn{2}{|c|}{$\begin{array}{l}\text { Within-group change at } \\
12 \text { months }\end{array}$} & \multirow{2}{*}{$\begin{array}{c}\text { Between-group } \\
\text { difference at } \\
12 \text { months }{ }^{\#}\end{array}$} \\
\hline & Usual care & Intervention & Usual care & Intervention & & Usual care & Intervention & \\
\hline
\end{tabular}

Data are presented as mean \pm SD or mean $\left(95 \%\right.$ CI), unless otherwise stated. Usual care $n=115$; intervention $n=157 .{ }^{\#}$ : adjusted for clustering, age, highest education, gross income, current smoking status, and existing chronic obstructive pulmonary disease; ${ }^{\uparrow}: n=258$ at baseline, $n=204$ at 6 months and $n=185$ at 12 months.

There were no significant differences in baseline characteristics between clinics that had at least one participant completing the full intervention versus clinics that had no participant completing the full intervention (supplementary file). Those participants who received the full intervention were more likely to be in a relationship and had higher SGRQ and CAT scores (i.e. lower quality of life) compared to those who did not receive the full intervention (supplementary file).

\section{Exploratory per protocol analysis}

An exploratory per protocol analysis was performed to assess the effect of the intervention in those who received the full intervention as intended. Statistically and clinically significant differences in change in SGRQ score at 6 months were observed within the intervention group (5.66, 95\% CI 1.91-9.42) and when compared to usual care (adjusted mean difference 5.22, 95\% CI 0.19-10.25; $\mathrm{p}=0.042$ ) (supplementary table S2). The improvement in SGRQ score in the intervention group was more pronounced and sustained at 12 months; the between-group difference was clinically, but not statistically significant. Multiple imputation analyses confirmed these patterns (supplementary table S3). More than half (23 (55\%) out of 42) of the full intervention group participants improved their SGRQ by the MCID or greater at 6 months (data not shown). Although changes in CAT score were more pronounced in the intervention group (at 6 and 12 months), between-group differences in CAT scores and FEV1 \% pred (at 6 months only) were not statistically significant.

\section{Discussion}

Our results showed no significant evidence for the effectiveness of an interdisciplinary model of care in primary care involving smoking cessation support, HMR and home-based pulmonary rehabilitation beyond usual care at improving HRQoL, symptom severity or lung function in a cohort of patients with predominantly mild COPD. Nevertheless, an improvement in mean SGRQ score was evident within the intervention group. Exploratory analyses of those who received the intended full intervention showed statistically and clinically significant differences over usual care at 6 months, and the benefits within the full intervention group were sustained at 12 months. However, uptake of the intended intervention components by both GPs and patients was suboptimal.

The effectiveness of integrated disease management programmes in COPD is still uncertain. While a recent Cochrane review showed positive effects of these interventions on HRQoL [7], there was considerable heterogeneity between the included studies, mainly due to differences in the healthcare systems where these trials took place [43]. Our interdisciplinary model coordinated by the patient's GP could not achieve fully integrated care due to a range of patient-, health professional- and health system-related factors. Our findings concur with recent studies of primary care-based COPD management programmes targeting those with relatively mild disease in Australia, the Netherlands and Switzerland, which showed no additional benefit of disease management and health professional partnership programmes on HRQoL over usual care [7-9].

More specifically, our results are similar to the primary care based COPD management programme of KRUIs et al. [7] conducted in the Netherlands; however, there were some key differences in the intervention design. KRUIs et al. offered an intervention consisting primarily of intensive training provided to GPs, nurses and physiotherapists on how to implement integrated disease management in practice, whereas RADICALS had a GP-coordinated interdisciplinary intervention where every GP and participant in the intervention arm was offered the intervention comprising smoking cessation support, HMR and home-based pulmonary rehabilitation. In addition, our study included a case-finding component for optimising the diagnosis of COPD in primary care. Our study population comprised many newly 
TABLE 3 COPD (chronic obstructive pulmonary disease) Assessment Test (CAT), lung function, modified Medical Research Council (mMRC) grade and Hospital Anxiety and Depression Scale (HADS) score differences from baseline to 6 and 12 months

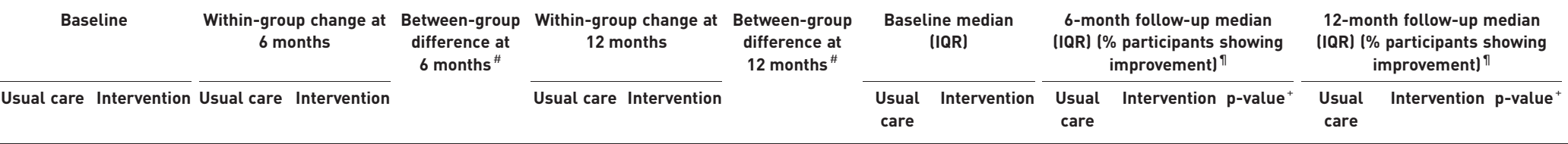

\begin{tabular}{|c|c|c|c|c|c|c|c|c|c|c|c|c|c|c|c|}
\hline CAT score ${ }^{\S}$ & $13.57 \pm 7.94$ & $12.94 \pm 7.57$ & $\begin{array}{l}1.50(0.56- \\
2.44)\end{array}$ & $\begin{array}{l}2.06(0.87- \\
3.26)\end{array}$ & $\begin{array}{l}0.66 \\
\quad(-1.98-3.30) \\
p=0.61\end{array}$ & $\begin{array}{l}2.62(1.58- \\
3.67)\end{array}$ & $\begin{array}{l}3.05(1.80- \\
4.31)\end{array}$ & $\begin{array}{l}0.86(-2.02- \\
3.74) p=0.55\end{array}$ & & & & & & & \\
\hline $\begin{array}{l}\text { Post-bronchodilator } \\
\text { FEV } \% \\
\text { predicted }^{f}\end{array}$ & $70.82 \pm 19.27$ & $68.98 \pm 20.46$ & $\begin{array}{l}-0.09 \\
\quad(-1.84- \\
1.67)\end{array}$ & $\begin{array}{c}0.79(-0.86- \\
2.44)\end{array}$ & $\begin{array}{l}1.09 \\
\quad(-1.59-3.76) \\
p=0.41\end{array}$ & NA & NA & NA & & & & & & & \\
\hline mMRC grade & & & & & & & & & $1(0-2)$ & $1(1-2)$ & $\begin{array}{c}1(1-2) \\
\quad(17.0)\end{array}$ & $1(1-2)(23.7) \quad 0.31$ & $\begin{array}{c}1(0-2) \\
\quad(18.2)\end{array}$ & $1(1-2)(21.2)$ & 0.74 \\
\hline $\begin{array}{l}\text { HADS anxiety } \\
\text { score }{ }^{\pi 17}\end{array}$ & & & & & & & & & $7(3-9)$ & $6(3-9)$ & $\begin{array}{c}3(0-6) \\
(69.8)\end{array}$ & $2(0-5)(70.8) 1.00$ & $\begin{array}{l}0(0-3) \\
\quad(87.1)\end{array}$ & $1(0-4)(80.0)$ & 0.32 \\
\hline $\begin{array}{l}\text { HADS depression } \\
\text { score }^{++}\end{array}$ & & & & & & & & & $4(2-7)$ & $5(2-7)$ & $\begin{array}{c}1(0-4) \\
(72.0)\end{array}$ & $1(0-4)(71.2) \quad 1.00$ & $\begin{array}{c}0(0-1.25) \\
(85.3)\end{array}$ & $\begin{array}{c}1(0-3.25) \\
(77.0)\end{array}$ & 0.30 \\
\hline
\end{tabular}

Data are presented as mean \pm SD or mean $(95 \% \mathrm{CI}$ ), unless otherwise stated. Usual care $\mathrm{n}=115$; intervention $\mathrm{n}=157$. IQR: interquartile range; FEV 1 : forced expiratory volume in $1 \mathrm{~s}$; NA: not available. ": adjusted for clustering, age, highest education, gross income, current smoking status and existing COPD; ": follow-up grade/score lower than baseline grade/score indicated improvement; ${ }^{+}: \mathrm{p}$-value of between-group differences in proportion of participants showing improvement in grade/score; ${ }^{\S}: \mathrm{n}=271$ at baseline, $\mathrm{n}=208$ at 6 months and $\mathrm{n}=189$ at 12 months; $^{f}: \mathrm{n}=269$ at baseline and $\mathrm{n}=185$ at 6 months; ${ }^{\# \#}: \mathrm{n}=271$ at baseline, $\mathrm{n}=208$ at 6 months and $\mathrm{n}=190$ at 12 months; "था: $n=264$ at baseline, $\mathrm{n}=186$ at 6 months and $\mathrm{n}=170$ at 12 months; ${ }^{++}: \mathrm{n}=264$ at baseline, $\mathrm{n}=190$ at 6 months and $\mathrm{n}=172$ at 12 months. 
diagnosed patients who had mild disease [13]. Low baseline SGRQ scores, low mMRC scores and mild disease based on FEV1 \% predicted [28] may explain the small effect observed. A "floor effect" is a possible explanation for the minimal changes observed in SGRQ, other quality-of-life scores and dyspnoea $[6,8,9]$. It is likely that COPD was not a priority for either GPs or participants for discussion during consultations and for subsequent interventions if patients had only mild symptoms, explaining the modest uptake of the interventions in this group.

Although the change in SGRQ in our trial was minimal, the magnitude of change in SGRQ in the intervention arm at 6 and 12 months is on a par with a recent large clinical trial of combination pharmacotherapies in patients with moderate to very severe COPD [44]. The interventions delivered in our study model are services or resources that are readily available and could be complementary to pharmacological treatments for COPD, if implemented early and more widely. This model could potentially be more effective in primary care by targeting those with symptoms and with better intervention fidelity.

Participation in pulmonary rehabilitation has been shown to improve HRQoL in patients with COPD [20]. The HomeBase programme in the RADICALS model is equivalent to centre-based pulmonary rehabilitation at improving HRQoL for patients with moderate to severe disease [22]. However, the effectiveness of pulmonary rehabilitation programmes for mild COPD is still uncertain [45]. The majority of our trial population had low levels of activity limitation (most participants self-reported low mMRC grades), and therefore may not have recognised the need for the intervention.

The roles of pharmacists in COPD management and positive effects of interventions on medication adherence and inhaler technique have been shown previously [18]. Such advice was routinely provided to participants by the pharmacist during the HMR. Due to the large number of practices and GPs involved, it was not possible to ensure that pharmacist recommendations were implemented by GPs, which may have diminished the effect.

\section{Strengths and limitations}

The key strength of this study was the assessment of real-world effectiveness of an interdisciplinary model of care comprising resources and services readily accessible in primary care. Multiple clinics and GPs were recruited into the study; clinics varied in size, availability of respiratory services and socioeconomic status of the clientele, increasing the generalisability of our findings. Cluster randomisation minimised the risk of contamination associated with the same practice staff treating participants from different trial arms. Although there was the potential for Hawthorne effect in this open-label study (participants and health professionals were not blinded), outcome assessments were performed by research assistants blinded to group allocation.

Practice and participant recruitment was challenging. The numbers of clinics and participants in the two arms were unbalanced and not all clinics contributed to the COPD cohort. Attrition rates were slightly higher than originally anticipated, also contributing to the small effects observed; those who completed all follow-ups may not be representative of the wider COPD population, especially those with mild or no symptoms. Greater variability in SGRQ scores was seen due to recruitment of subjects with any severity of COPD. We did not assess changes in pharmacological or non-pharmacological COPD management in usual care participants. In addition, we could not tell whether participants undertook traditional hospital outpatient pulmonary rehabilitation or received HMR (both existing services in Australia) during the study through other sources. Trial effects, dissemination of COPD-X and smoking cessation guidelines, lung function testing and provision of spirometry results to GPs in the usual care arm might have prompted changes in COPD management in these participants which would not have otherwise occurred. We did not assess changes in participant behaviours such as adherence to medication, inhaler technique and adherence to home exercises.

A key limitation is the low uptake of the intervention components. Due to logistical and resource limitations, we did not organise interviews with patients, practice managers or GPs to obtain more information on process outcomes and detailed reasons for the low uptake. Although per protocol analyses revealed statistically significant changes in SGRQ at 6 months, this standalone positive outcome should be interpreted with caution. Most importantly, poor uptake of the intervention does not allow us to interpret whether the negative findings were resultant from being unable to deliver the intervention or from intervention failure.

\section{Practice implications and future research}

Challenges in implementation and slow uptake of the non-pharmacological interventions offered within this pragmatic trial are important lessons for future primary care studies. There were delays in delivering 
the multi-component intervention due to the multiple steps involved, e.g. spirometry results review (with or without expert input in interpretation and diagnosis), organising of patient visits to discuss results, eventual referral to HMR and/or HomeBase by GP following patient consent and GP follow-up visit to implement pharmacist HMR report recommendations. Efforts made to educate GPs and participants on the potential benefits of HMR and HomeBase did not necessarily translate to referral and/or uptake. Although the clinic management agreed to participate in the trial, not all GPs practising within each clinic actively supported trial implementation. Limited resources for delivering the intervention at the clinic level due to competing demands for GPs' time and inadequate remuneration for delivering the service might have been barriers. Lack of implementation fidelity can make interventions appear to be ineffective [46]

Future studies should factor in the real-world challenges of recruitment, time considerations, and diagnostic and referral processes in the primary care setting. Specific training on integrated disease management concepts provided to health professionals involved should be considered and may potentially increase delivery of integrated care in practice.

Where possible, a phased approach to intervention development with preliminary testing (including collecting information on reasons for non-referral to intervention components) should be conducted to inform feasibility and level of uptake of interventions by GPs and participants. Engagement of individual GPs, a more proactive and streamlined intervention referral process, detailed explanation of the disease and its effects to patients, and the potential benefits of non-pharmacological interventions such as HomeBase and HMRs may improve participant interest and intervention uptake.

In primary care, uptake of and adherence to healthy behaviours (smoking cessation, increased physical activity) and self-management skills (optimal use of inhaled medications and early recognition and treatment of worsening of symptoms) may be better predictors of longer term outcomes in patients with mild disease. Patient needs, preferences and personal goals should be assessed carefully and considered to inform subsequent intervention programme tailoring.

Acknowledgements: We wish to thank Data Safety and Monitoring Board members (Ian Yang, Jennifer Alison and Julia Walters), Denise van den Bosch, Jessica Webster, Rita Breare, Mieke Hutchinson-Kern, Brigitte Borg and The Alfred Respiratory Laboratory, Eleonora Del Colle and Pulmetrics staff, Brian Meier, Eli Dabscheck, Agape Stratigis, Amanda Cross, Amanda Nichols, Andrew Clayton, Caroline Nicolson, Francesca Sgroi, Georgia Lakoumentas, Hilary Edwards, Janet Carberry, Jannette Angell, Kay Dunkley, Lisa Austin, Melanie Frodsham, Robyn Saunders, Robyn Stell, Ross de Gori, Rosemary Moore, Sandy Scholes, Sarah Rawlings, Tamara Ford, Yamuna Prashanth, research students, clinic staff and participants.

Author contributions: J. George conceived the research idea and developed it with input from chief investigators. M.J. Abramson, G. Russell, N.A. Zwar, A.E. Holland, B. Bonevski and A. Mahal. Representatives of partner organisations (K. Phillips and P. Eustace) contributed to discussions. J. Liang is a $\mathrm{PhD}$ student working under the supervision of M.J. Abramson and J. George. J. Liang coordinated data collection and data management. E. Paul and J. Liang carried out statistical analyses required for the manuscript. S. Wilson was the project manager responsible for the recruitment of clinics and the overall conduct of the study. N.S. Cox coordinated delivery of the HomeBase programme. J. Liang drafted the original manuscript draft. All authors contributed to data interpretation and manuscript revision, and provided approval of the final manuscript.

Conflict of interest: M.J. Abramson reports grants from Boehringer Ingelheim, during the conduct of the study; grants from Pfizer, assistance with conference attendance and personal fees for consultancy from Sanofi, outside the submitted work. G. Russell has nothing to disclose. A.E. Holland is a current member of the Lung Foundation Australia COPD-X: Concise Guide for Primary Care Advisory Committee. N.A. Zwar is a current member of the Lung Foundation Australia COPD Guidelines Committee. B. Bonevski has nothing to disclose. A. Mahal has nothing to disclose. P. Eustace has nothing to disclose. E. Paul has nothing to disclose. K. Phillips is the Lung Foundation Australia General Manager of Consumer Programs. The Lung Foundation Australia works in collaboration and receives funding from pharmaceutical companies outlined in the foundation's annual reports (available at lungfoundation.com.au/about-us/annual-reports/). N.S. Cox has nothing to disclose. S. Wilson has nothing to disclose. J. George reports grants from Boehringer Ingelheim, during the conduct of the study; grants from Pfizer, and personal fees for consultancy from GSK, outside the submitted work; and is a current member of the Lung Foundation Australia COPD Guidelines Committee. J. Liang has nothing to disclose.

Support statement: We wish to thank all our funding bodies and partner organisations for supporting the trial (Boehringer Ingelheim, Eastern Melbourne PHN, Lung Foundation Australia and National Health and Medical Research Council). Lung Foundation Australia and Eastern Melbourne PHN were involved in project design and conduct, and contributed to data interpretation and writing of manuscripts. Boehringer Ingelheim was involved in project discussions, planning and progress review, but had no involvement in the design of the intervention programme and did not contribute to decisions regarding data analysis and dissemination of findings. B. Bonevski is supported by an Australian National Health and Medical Research Council Career Development Fellowship (GNT1063206) and a Faculty of Health and Medicine, University of Newcastle Gladys M Brawn Career Development Fellowship. J. Liang is the recipient of the Cyril Tonkin Scholarship 2014 (Faculty of Pharmacy and Pharmaceutical Sciences Foundation Board, Monash University). Funding information for this article has been deposited with the Crossref Funder Registry. 


\section{References}

1 Schermer T, van Weel C, Barten F, et al. Prevention and management of chronic obstructive pulmonary disease (COPD) in primary care: position paper of the European Forum for Primary Care. Qual Prim Care 2008; 16: 363-377.

2 Victorian Government Department of Health Services. Health Independence Program Guidelines. Melbourne, Victorian Government Department of Health Services, 2008.

3 Jessup RL. Interdisciplinary versus multidisciplinary care teams: do we understand the difference? Aust Health Rev 2007; 31: 330-331.

4 Schrijvers G. Disease management: a proposal for a new definition. Int J Integr Care 2009; 9: e06.

5 Kruis AL, Smidt N, Assendelft WJ, et al. Integrated disease management interventions for patients with chronic obstructive pulmonary disease. Cochrane Database Syst Rev 2013; CD009437.

6 Zwar NA, Hermiz O, Comino E, et al. Care of patients with a diagnosis of chronic obstructive pulmonary disease: a cluster randomised controlled trial. Med J Aust 2012; 197: 394-398.

7 Kruis AL, Boland MR, Assendelft WJ, et al. Effectiveness of integrated disease management for primary care chronic obstructive pulmonary disease patients: results of cluster randomised trial. BMJ 2014; 349: g5392.

8 Markun S, Rosemann T, Dalla-Lana K, et al. Care in Chronic Obstructive Lung Disease (CAROL): a randomised trial in general practice. Eur Respir J 2018; 51: 1701873.

9 Zwar NA, Bunker JM, Reddel HK, et al. Early intervention for chronic obstructive pulmonary disease by practice nurse and GP teams: a cluster randomized trial. Fam Pract 2016; 33: 663-670.

10 Price D, West D, Brusselle G, et al. Management of COPD in the UK primary-care setting: an analysis of real-life prescribing patterns. Int J Chron Obstruct Pulmon Dis 2014; 9: 889-904.

11 Bourbeau J, Sebaldt RJ, Day A, et al. Practice patterns in the management of chronic obstructive pulmonary disease in primary practice: the CAGE study. Can Respir J 2008; 15: 13-19.

12 Jones RC, Dickson-Spillmann M, Mather MJ, et al. Accuracy of diagnostic registers and management of chronic obstructive pulmonary disease: the Devon primary care audit. Respir Res 2008; 9: 62.

13 Liang J, Abramson MJ, Zwar NA, et al. Diagnosing COPD and supporting smoking cessation in general practice: evidence-practice gaps. Med J Aust 2018; 208: 29-34.

14 Lundbäck B, Lindberg A, Lindström M, et al. Not 15 but 50\% of smokers develop COPD? - report from the Obstructive Lung Disease in Northern Sweden studies. Respir Med 2003; 97: 115-122.

15 George J, Kong DC, Thoman R, et al. Factors associated with medication nonadherence in patients with COPD. Chest 2005; 128: 3198-3204.

16 Lareau SC, Yawn BP. Improving adherence with inhaler therapy in COPD. Int J Chron Obstruct Pulmon Dis 2010; 5: 401-406.

17 Hesso I, Gebara SN, Kayyali R. Impact of community pharmacists in COPD management: inhalation technique and medication adherence. Respir Med 2016; 118: 22-30.

18 van der Molen T, van Boven JF, Maguire T, et al. Optimizing identification and management of COPD patients reviewing the role of the community pharmacist. Br J Clin Pharmacol 2017; 83: 192-201.

19 Brooks D, Sottana R, Bell B, et al. Characterization of pulmonary rehabilitation programs in Canada in 2005. Can Respir J 2007; 14: 87-92.

20 McCarthy B, Casey D, Devane D, et al. Pulmonary rehabilitation for chronic obstructive pulmonary disease. Cochrane Database Syst Rev 2015; CD003793.

21 Yohannes AM, Connolly MJ. Pulmonary rehabilitation programmes in the UK: a national representative survey. Clin Rehabil 2004; 18: 444-449.

22 Holland AE, Mahal A, Hill CJ, et al. Home-based rehabilitation for COPD using minimal resources: a randomised, controlled equivalence trial. Thorax 2017; 72: 57-65.

23 Liang J, Abramson MJ, Zwar N, et al. Interdisciplinary model of care (RADICALS) for early detection and management of chronic obstructive pulmonary disease (COPD) in Australian primary care: study protocol for a cluster randomised controlled trial. BMJ Open 2017; 7: e016985.

24 Lung Foundation Australia. 2018. Position Paper: COPD Case Finding in Community Settings. https:// lungfoundation.com.au/wp-content/uploads/2018/11/Information-paper-COPD-Case-Finding-position-paper-Oct2018. pdf.

25 Frith P, Crockett A, Beilby J, et al. Simplified COPD screening: validation of the PiKo- $6^{\oplus}$ in primary care. Prim Care Respir J 2011; 20: 190-198.

26 Miller MR, Hankinson J, Brusasco V, et al. Standardisation of spirometry. Eur Respir J 2005; 26: 319-338.

27 Yang IA, Brown JL, George J, et al. COPD-X Australian and New Zealand guidelines for the diagnosis and management of chronic obstructive pulmonary disease: 2017 update. Med J Aust 2017; 207: 436-442.

28 Yang IA, Dabscheck E, George J, et al. The COPD-X Plan: Australian and New Zealand Guidelines for the Management of Chronic Obstructive Pulmonary Disease 2017. Version 2.52. https://copdx.org.au/copd-x-plan/ Date last accessed April 5 2018. Date last updated December 2017.

29 Zwar N, Richmond R, Borland R, et al. Supporting Smoking Cessation: a Guide for Health Professionals. Melbourne, The Royal Australian College of General Practitioners, 2011.

30 Lung Foundation Australia. Better Living with Chronic Obstructive Pulmonary Disease: A Patient Guide. 2nd edn Queensland, The State of Queensland (Queensland Health) and Lung Foundation Australia, 2012.

31 Thomas D, Abramson MJ, Bonevski B, et al. A pharmacist-led system-change smoking cessation intervention for smokers admitted to Australian public hospitals (GIVE UP FOR GOOD): study protocol for a randomised controlled trial. Trials 2013; 14: 148

32 Pharmaceutical Society of Australia. Guidelines for Pharmacists Providing Home Medicines Review (HMR) Services 2011. https://aacp.com.au/app/uploads/home-medicines-review-services-1.pdf

33 Jones PW, Quirk FH, Baveystock CM, et al. A self-complete measure of health status for chronic airflow limitation. The St George's Respiratory Questionnaire. Am Rev Respir Dis 1992; 145: 1321-1327.

34 Jones PW, Harding G, Berry P, et al. Development and first validation of the COPD Assessment Test. Eur Respir J 2009; 34: 648-654

35 Celli BR, Cote CG, Marin JM, et al. The body-mass index, airflow obstruction, dyspnea, and exercise capacity index in chronic obstructive pulmonary disease. N Engl J Med 2004; 350: 1005-1012. 
36 Mahler DA, Wells CK. Evaluation of clinical methods for rating dyspnea. Chest 1988; 93: 580-586.

37 Quanjer PH, Stanojevic S, Cole TJ, et al. Multi-ethnic reference values for spirometry for the 3-95-yr age range: the global lung function 2012 equations. Eur Respir J 2012; 40: 1324-1343.

38 Snaith RP. The hospital anxiety and depression scale. Health Qual Life Outcomes 2003; 1: 29.

39 Zigmond AS, Snaith RP. The hospital anxiety and depression scale. Acta Psychiatr Scand 1983; 67: 361-370.

40 Heatherton TF, Kozlowski LT, Frecker RC, et al. Measuring the heaviness of smoking: using self-reported time to the first cigarette of the day and number of cigarettes smoked per day. Br J Addict 1989; 84: 791-799.

41 West R, Hajek P, Stead L, et al. Outcome criteria in smoking cessation trials: proposal for a common standard Addiction 2005; 100: 299-303.

42 Adams G, Gulliford MC, Ukoumunne OC, et al. Patterns of intra-cluster correlation from primary care research to inform study design and analysis. J Clin Epidemiol 2004; 57: 785-794.

43 Kruis AL, Smidt N, Assendelft WJ, et al. Cochrane corner: is integrated disease management for patients with COPD effective? Thorax 2014; 69: 1053-1055.

44 Lipson DA, Barnhart F, Brealey $\mathrm{N}$, et al. Once-daily single-inhaler triple versus dual therapy in patients with COPD. N Engl J Med 2018; 378: 1671-1680.

45 Alison JA, McKeough ZJ, Johnston K, et al. Australian and New Zealand Pulmonary Rehabilitation Guidelines. Respirology 2017; 22: 800-819.

46 Breitenstein SM, Gross D, Garvey CA, et al. Implementation fidelity in community-based interventions. Res Nurs Health 2010; 33: 164-173. 